\title{
Congestion Pricing Optimization for Shared Transport
}

\author{
Andres Osuna \& Hongcheng Liu \\ University of Florida
}

Faculty Mentor: Hongcheng Liu, Industrial and Systems Engineering

\begin{abstract}
Toll pricing has long been recognized as an effective approach to manage traffic congestion. This project includes the analysis of transportation systems by mathematically modeling the impact of pricing on the collective decision-making of drivers via Nash games. The objective is to develop an optimal pricing strategy that maximizes shared transport companies' revenue while minimizing drivers' time costs. A Nash game theoretic framework is used to tackle the long-term challenge on how to properly capture the nonlinear nature of traffic users' behavior. Two types of traffic pricing models were developed; a base case with a fixed demand and a more realistic case using elastic demand. The base case was established for users who vary their routing choices but not their transportation modes. The second model is more appropriate for companies with users who optimize both their routing and choice of transportation based on the pricing policy of the system. Optimal pricing policies can be determined by convex formulations of optimal system traffic assignment problems on simple networks with different origins and destinations. The numerical examples show formulation feasibility and provide insight on how the company can have a positive impact on both its revenue and users' time cost by modifying its pricing strategy. The models also show how pricing affects road users' decisions under different parameter settings. The paper demonstrates that a shared transport company can increase revenue by determining key links and properly capturing users' behavior while reaching the maximum level of revenue over time cost ratio

Keywords: Nash games, shared transport, traffic assignment, pricing optimization, driver's decision-making
\end{abstract}

\section{Introduction}

Toll pricing has long been recognized as one of the best methods to improve traffic flow and congestion in urban networks. This method is also applied by shared transport companies for pricing their services. Nowadays, real-time shared transport companies gather data to revolutionize the transportation industry for a more centered and optimized transportation structure (Bertsimas et al., 2019). A challenge in pricing shared transport stems from the nonlinear nature of traffic users' behavior as a response to the deployed pricing scheme. To better capture this, the models in this paper were developed while adopting Nash games as a framework. By using this theory, the model searches for a user equilibrium that can satisfy traffic system constraints. The Nash games in this setting consist of $n+m$ players in a non-cooperative system where $\mathrm{n}$ users search their best routes in a network with $\mathrm{m}$ origin-destination pairs. This 
framework will allow the models to better simulate the players' strategy changing behavior. If the company has selected a strategy, mantains it, and the network users do not improve their objective by modifying their strategies, then the current solution set constitutes a Nash equilibrium.

There have been some proposed bilevel programming approaches to determine toll levels on predetermined links without capacity constraints, using both elastic and fixed demand (Yang \& Zang, 2003). Congestion pricing models have suggested that the drivers' strategic routes modifications do contribute to congestion reduction in traveling with lower price levels (May \& Milne, 2000). While taking into account queueing and congestion conditions, other exploratory bilevel programming approaches have also been made (Yan \& Lam, 1996). Modifications of these models, while considering multiclass within the pool of network users, were also developed to capture different economic status within the users (Yang \& Zang, 2002). These are not sufficient to completely capture all classes within the network since drivers' economic status tend to be already addressed by the choice of transportation mode. These publications based their framework on the idea that there is a lead player and then the followers, which does not reflect users' real behavior in this system. The upper level of their models aimed to minimize total network travel time while the lower level aims to simulate the route choice behavior of all users.

Those ideas can also be modified and implemented to verify if shared transport companies can maximize their revenue while improving social welfare. This paper shows the development of a Nash equilibrium problem in which the overall objective of the game is to determine optimal link price levels and flows of the system. It examines a model formulation for fixed and elastic demand. The first player's objective is to maximize revenue while the second player aims to minimize the total network's users cost.

There is a lot of additional background research on traffic equilibrium assignment and the calculation of link costs as functions of the link flows (Bell \& Cassir, 2002). Other authors have used pricing models for modeling multimodal traffic with focus on parking pricing strategies (Zheng \& Geroliminis, 2016). Continuous studies have been made about the application of game theory into a risk-averse equilibrium traffic assignment formulation problem (Bell \& Cassir, 2002).

For better understanding of the models, it is important to present all variables and parameters taken into consideration in this problem (Table 1). 
Table 1. Model notation

\begin{tabular}{|c|c|c|}
\hline Variables \& Parameters & Units & Description \\
\hline A & - & Set of links \\
\hline $\mathrm{W}$ & - & Original-destination (O-D) pairs \\
\hline M & - & Set of transportation class \\
\hline$R_{w}$ & - & Set of paths (O-D), $w \in W$ \\
\hline$P_{k}$ & - & Term priority coefficient $(\mathrm{k}=1,2)$ \\
\hline$\tau$ & - & Time value coefficient \\
\hline$\delta_{a r}$ & - & $\begin{array}{c}=1 \text { if route } \mathrm{r} \text { uses link a } \\
\quad=0 \text { if otherwise }\end{array}$ \\
\hline$\gamma_{r w}$ & - & $\begin{array}{c}=1 \text { if route } \mathrm{r} \text { is between } \mathrm{O}-\mathrm{D} \text { pair } \mathrm{W} \\
=0 \text { if otherwise }\end{array}$ \\
\hline$v_{a}^{m}$ & veh/hr. & Flow on link a of class $\mathrm{m}$ \\
\hline$f_{r}^{m}$ & veh/hr. & Traffic flow on route $r$ of class $m$ \\
\hline$q_{w}^{m}$ & veh/hr. & Fixed demand for each (O-D) pair by class $m$ \\
\hline$y_{a}^{m}$ & $\$$ & Price charged by class $\mathrm{m}$ and link a \\
\hline$d_{w}^{m}$ & veh/hr. & (O-D) demand variable by class $\mathrm{m}$ \\
\hline
\end{tabular}

(Yang \& Zhang, 2003)

The following section examines the game-theoretic models for pricing scheme optimization. Later, numerical examples are solved with a simple programming approach using Generic Algebraic Modeling System (GAMS). A final section with further analysis of the results, demonstrating the feasibility of the methods and models developed is provided. Conclusions are presented in the final section.

\section{Determination of optimal price levels on predetermined links}

Let $\mathrm{G}(\mathrm{N}, \mathrm{A})$ be a network defined by a set of nodes $(\mathrm{N})$ connected by a set of directed links (A). Also, let $t_{a}^{m}\left(v_{a}^{m}, v_{a}^{\widetilde{m}}, \widetilde{m} \neq m\right)$ be a continuous, convex and strictly increasing function of all flows $v_{a}^{m}$ (Yang $\&$ Zhang, 2003). This function represents the total travel time per choice of transportation $\mathrm{m} \epsilon M$ and link a $\epsilon A$ used. Then, as shown in eq. F1, this function is composed by three terms. Let $\alpha_{a}^{m}$ be the free flow travel time in link a $\epsilon A$ by transportation mode $\mathrm{m} \epsilon M$. Furthermore, it is important to consider the impact of congestion of the entire flow $v_{a}^{m}$ on the rest of the drivers. Thus, $\beta_{a}^{m}$ will be the time-congestion impact coefficient on link a $\epsilon A$ by class $\mathrm{m} \in M$. In addition, assume all types of transportation affect each other. 
Then, as shown in eq. F2, the overall link usage cost will be a dependent function $C_{a}^{m}\left(t_{a}^{m}, y_{a, m}\right)$, which is the sum of both monetary and time cost. Therefore, eq. F2 defines a function composed by $\mathrm{F} 1$ and the price charged $y_{a, m}$.

$$
\begin{gathered}
t_{a}^{m}\left(v_{a}^{m}, v_{a}^{\widetilde{m}}, \tilde{m} \neq m\right)=\alpha_{a}^{m}\left(1+\beta_{a}^{m} v_{a}^{m}\right)+\sum_{\tilde{m} \neq m} \beta_{a}^{\widetilde{m}} \alpha_{a}^{\widetilde{m}} v_{a}^{\widetilde{m}} \\
C_{a}^{m}\left(v_{a}^{m}, y_{a}^{m} ; v_{a}^{\widetilde{m}}, \widetilde{m} \neq m\right)=\tau * t_{a}^{m}\left(v_{a}^{m}, v_{a}^{\widetilde{m}}, \widetilde{m} \neq m\right)+y_{a}^{m}
\end{gathered}
$$

To develop the upcoming models, bilevel programs that determined system optimal toll levels while considering link capacity constraints were examined (Yan \& Lam, 1996). This model could be adapted into a Nash game formulation, in which the shared transport company and the users are players of a system. Moreover, this paper is not considering link capacity constraints since $\beta_{a}^{m}$ already takes into account the congestion impact on the initial link traveled time. Finally, the models are formulated from the perspective of the company and users of the network.

\section{Model A}

The first model is formulated considering fixed demand across all (O-D) pairs and transportation modes. The objectives of this model are to capture the company's (player 1) and network users' (player 2) behavior.

player 1 strategy formulation. The first section represents the company's objective and constraints:

$$
\begin{gathered}
\max _{y, v, f} \sum_{a \in A} \sum_{m=1}^{M} y_{a}^{m} * v_{a}^{m}(y) \\
\text { S.T. } y_{a, m}^{\min } \leq y_{a, m} \leq y_{a, m}^{\max } \quad \forall a \in A, \forall m \in M
\end{gathered}
$$

These equations represent the objective and systematic boundaries of player 1 (company). The above pricing policy is under a single constraint, defined by a lower bound $y_{a, m}^{\min }$ and upper bound $y_{a, m}^{\max }(2)$, representing the assumption that there are market regulations to the price charged by shared transport companies. Here $v_{a}^{m}(y)$ is the solution of the following parallel program (player 2's strategy).

player 2 strategy formulation. This represents the users' equilibrium problem response to the company's pricing policy decision (player 1's strategy). 


$$
\begin{gathered}
\min _{v, f} \sum_{a \in A} \sum_{m=1}^{M} \int_{0}^{v_{a}^{m}} C_{a}^{m}\left(\theta, y_{a}^{m} ; v_{a}^{\tilde{m}}, \tilde{m} \neq m\right) d \theta \\
\sum_{r \in R_{w}} f_{r}^{m} * \gamma_{r w}=q_{w}^{m} \quad \forall w \in W, \forall m \in M \\
v_{a}^{m}=\sum_{r \in R_{w}} f_{r}^{m} * \delta_{a r} \quad \forall a \in A, \forall m \in M \\
f_{r}^{m} \geq 0 \quad \forall r \in R_{w}, \forall m \in M
\end{gathered}
$$

This section of the model formulates the objective of minimizing overall users' total travel time cost eq. F2 (3). The model guarantees that all fixed demand will be satisfied (4), by using $\gamma_{r w}$ to define the relationship between routes and all O-D pairs. Constraint (5) defines the objective function's variable $v_{a}^{m}$ by determining the link flow from the route flow $f_{r}^{m}$. It also uses $\delta_{a r}$ to determine which links are part of each route. This will determine the optimal user flow for each link a $\epsilon A$.

As mentioned before, Model A is formulated using fixed demand. To include demand elasticity, it is important to remember that both supply and demand must be considered (Yang \& Zhang, 2003). They developed another model using a bilevel programming approach that could find the optimal price levels for tolls considering elasticity (Yang \& Zhang, 2002).

\section{Model B}

In the next model, the shared transport company (player 1) is considering demand elasticity. Therefore, this model is formulated considering a variable demand across all (O-D) pairs and transportation modes.

player 1 strategy formulation. The first section represents the company's objective and constraints:

$$
\begin{gathered}
\max _{y, d, v, f} P_{1} \sum_{w \in W} \sum_{m=1}^{M} \int_{0}^{d_{w}^{m}(y)} D_{w}^{m^{-1}}(\omega) d \omega+P_{2} \sum_{a \in A} \sum_{m=1}^{M} y_{a}^{m} * v_{a}^{m}(y) \\
\text { S.T. } y_{a, m}^{\min } \leq y_{a, m} \leq y_{a, m}^{\max } \quad \forall a \in A, \forall m \in M
\end{gathered}
$$

The objective function (7) aims to maximize the revenue while considering the demand function $D_{w}^{m}\left(\mu_{w}^{m}\right)$ for each O-D pair $w \in W . D_{w}^{m}\left(\mu_{w}^{m}\right)$ is a strictly increasing, convex and continuous function of the cost $\mu_{w}^{m}$ (Yang \& Zhang, 2002). Therefore, $D_{w}^{m^{-1}}\left(d_{w}^{m}\right)$ defines the 
inverse of the demand function; the lower the cost, the more demand the company will get, but at the same time, the less revenue it might generate. To further develop the relationship between these two terms, the objective function also contains $P_{k}(\mathrm{k}=1,2)$ which gives the company the flexibility to give more priority to a term than the other. Here $v_{a}^{m}(y)$ and $d_{w}^{m}(y)$ are the solutions of the following parallel program (player 2 strategy).

player 2 strategy formulation. This represents the users' equilibrium response to the company's pricing policy decision (player 1's strategy).

$$
\begin{gathered}
\min _{d, v, f} \sum_{a \in A} \sum_{m=1}^{M} \int_{0}^{v_{a}^{m}} C_{a}^{m}\left(\theta, y_{a}^{m} ; v_{a}^{\widetilde{m}}, \tilde{m} \neq m\right) d \theta-\sum_{w \in W} \sum_{m=1}^{M} \int_{0}^{d_{w}^{m}} D_{w}^{m^{-1}}(\omega) d \omega \\
\text { S. T. } \\
\sum_{r \in R_{w}} f_{r}^{m} * \gamma_{r w}=d_{w}^{m} \quad \forall w \in W, \forall m \in M \\
v_{a}^{m}=\sum_{r \in R_{w}} f_{r}^{m} \delta_{a r} \quad \forall a \in A, \forall m \in M \\
f_{r}^{m} \geq 0 \quad \forall r \in R_{w}, \forall m \in M
\end{gathered}
$$

This entire formulation uses the same boundaries as the player 2's strategy section in Model A, but the objective function (9) represents the user equilibrium of the system, which balances the cost per demand function $D_{w}^{m^{-1}}$ and the total cost of the users.

\section{Numerical Examples}

All networks are composed by nodes connected by links with a certain demand for each pair of nodes. The model allows for drivers to make decisions to minimize the total cost of network usage. The users' strategy section for Model A was tested for two different network examples and found interesting feasible results that will have an impact on how transportation users behave in response to different pricing policies.

\section{Simple Network- Example 1}

For this example, the network is presented in Figure 1 and the problem formulation is solved using all specified parameters in Model A. The results were obtained using a predetermined pricing policy as seen in Table 2 . Both objective function parameters $\left(\beta_{a}^{m}\right.$ and $\left.\alpha_{a}^{m}\right)$ values are shown in Table 3 and 4, respectively. Finally, the demand for each O-D pair is shown in Table 5. 


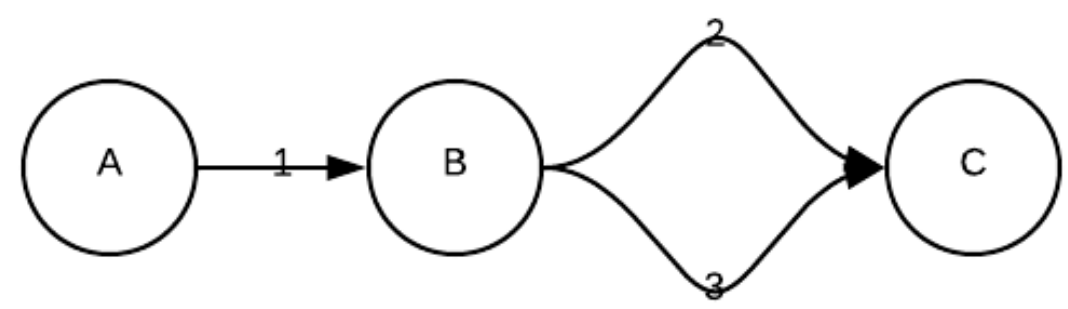

Figure 1.Simple Network for fixed demand problem.

Table 2. Pricing policy input for example 1

\begin{tabular}{lccc}
\hline$y_{m, a}$ & 1 & 2 & 3 \\
\cline { 2 - 4 } Car & $\$ 2.50$ & $\$ 1.70$ & $\$ 3.10$ \\
Bicycle & $\$ 0.50$ & $\$ 0.60$ & $\$ 0.80$ \\
Scooter & $\$ 1.10$ & $\$ 1.20$ & $\$ 1.10$ \\
\hline
\end{tabular}

Table 3. Time impact coefficients for example 1

\begin{tabular}{lccc}
\hline$\beta_{a}^{m}$ & 1 & 2 & 3 \\
\hline Car & 0.11 & 0.09 & 0.11 \\
Bicycle & 0.09 & 0.08 & 0.07 \\
Scooter & 0.10 & 0.16 & 0.12 \\
\hline
\end{tabular}

Table 4. Free flow travel time in min for example 1

\begin{tabular}{llll}
\hline$\alpha_{a}^{m}$ & 1 & 2 & 3 \\
\hline Car & 4 & 3 & 5 \\
Bicycle & 6 & 4 & 6 \\
Scooter & 8 & 7 & 8 \\
\hline
\end{tabular}

Table 5. Demand for example 1

\begin{tabular}{lccc}
\hline$q_{w}^{m}$ & $\mathrm{~A}-\mathrm{B}$ & $\mathrm{A}-\mathrm{C}$ & $\mathrm{B}-\mathrm{C}$ \\
\hline Car & 20 & 30 & 35 \\
Bicycle & 5 & 6 & 4 \\
Scooter & 10 & 8 & 12 \\
\hline
\end{tabular}

This example served as a modeling proof to make sure the model is feasible and can produce tangible results. As seen in Figure 1 and Table 5, the entire demand from A to B will be satisfied using link 1. The problem becomes interesting for O-D pairs A-C and B-C since the network splits in two different routes for each pair. 
Table 6. Example 1 results

\begin{tabular}{lccc}
\hline$v_{a}^{m}$ & 1 & 2 & 3 \\
\hline Car & 50 & 43 & 22 \\
Bicycle & 11 & 6 & 4 \\
Scooter & 18 & 10 & 10 \\
\hline
\end{tabular}

The system optimum flows per link and mode are shown in Table 6. This proves that the entire demand is met and that there is a preference for cheaper links. However, the users are not all employing the same link because they understand that it will cost them more time in congestion. For this example, $\tau$ will be equal to $\$ 15$ for the calculation of users' time cost which resulted on a total of $\$ 1,400$ per hour, and a revenue of $\$ 320$ per hour. Having such a small revenue per hour makes sense because of the size of the network.

\section{Intermediate Network- Example 2}

This paper examines a second numerical experiment on a more complicated network as seen in Figure 2. This network consists of an extension of the previous example, having 6 links and 4 different nodes, making this problem more complex than example 1. Since this is an extension of example 1, some of the parameters stayed the same. This can be seen from Table 7 to 10 .

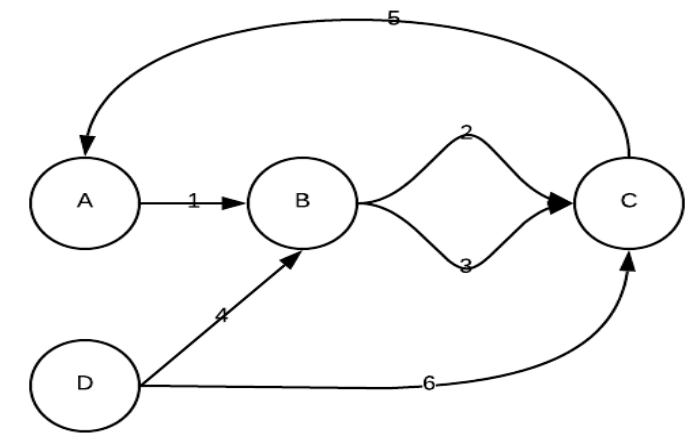

Figure 2. Intermediate network for fixed demand problem.

Table 7. Pricing policy input for example 2

\begin{tabular}{lcccccc}
\hline$y_{m, a}$ & 1 & 2 & 3 & 4 & 5 & 6 \\
\hline Car & $\$ 2.50$ & $\$ 1.70$ & $\$ 3.10$ & $\$ 2.2$ & $\$ 2.80$ & $\$ 2.00$ \\
Bicycle & $\$ 0.50$ & $\$ 0.60$ & $\$ 0.80$ & $\$ 0.6$ & $\$ 0.50$ & $\$ 0.40$ \\
Scooter & $\$ 1.10$ & $\$ 1.20$ & $\$ 1.10$ & $\$ 1$ & $\$ 1.20$ & $\$ 0.90$ \\
\hline
\end{tabular}

Table 8. Time impact coefficients for example 2 


\begin{tabular}{lcccccc}
\hline$\beta_{a}^{m}$ & 1 & 2 & 3 & 4 & 5 & 6 \\
\hline Car & 0.11 & 0.09 & 0.11 & 0.10 & 0.13 & 0.12 \\
Bicycle & 0.09 & 0.08 & 0.07 & 0.05 & 0.04 & 0.05 \\
Scooter & 0.10 & 0.16 & 0.12 & 0.10 & 0.09 & 0.11 \\
\hline
\end{tabular}

Table 9. Free flow travel time in min for example 2

\begin{tabular}{lllllll}
\hline$\alpha_{a}^{m}$ & 1 & 2 & 3 & 4 & 5 & 6 \\
\hline Car & 4 & 3 & 5 & 2 & 4 & 6 \\
Bicycle & 8 & 7 & 8 & 6 & 7 & 9 \\
Scooter & 6 & 4 & 6 & 5 & 4 & 6 \\
\hline
\end{tabular}

Table 10. Demand for example 2

\begin{tabular}{lccccccccc}
\hline$q_{w}^{m}$ & A-B & A-C & B-C & B-A & C-A & C-B & D-A & D-B & D-C \\
& & & & & & & & & \\
\hline Car & 20 & 30 & 35 & 20 & 15 & 12 & 10 & 15 & 25 \\
Bicycle & 5 & 6 & 4 & 3 & 4 & 5 & 5 & 5 & 6 \\
Scooter & 10 & 8 & 12 & 8 & 1 & 8 & 6 & 7 & 9 \\
\hline
\end{tabular}

For the intermediate network, the feasibility of the problems stands, and some interesting system optimum flow links can be seen in Table 11 .

\begin{tabular}{lcccccc} 
Table 11. Example 2 results \\
\hline$v_{a}^{m}$ & 1 & 2 & 3 & 4 & 5 & 6 \\
\hline Car & 62 & 55 & 30 & 15 & 57 & 35 \\
Bicycle & 16 & 8 & 5 & 5 & 17 & 11 \\
Scooter & 26 & 11 & 10 & 7 & 32 & 15 \\
\hline
\end{tabular}

This proves once again that the entire demand is satisfied, and that there is a preference for cheaper links. For example, in this case, users who are planning to go from D to A would rather use link 6 over 4 because it saves them having to go through an extra link that heavily increases their cost. For this example, $\tau$ is also used as $\$ 15$ for the calculation of time cost and resulted on a total of $\$ 3,946$ per hour, and a revenue of $\$ 748$ per hour. 
Table 12. Example 2 iteration results

\begin{tabular}{|c|c|c|c|c|c|c|c|c|c|}
\hline Iteration & $y_{m, a}$ & 1 & 2 & 3 & 4 & 5 & 6 & $\begin{array}{c}\text { Time cost } \\
(\$ / \mathrm{hr} .)\end{array}$ & $\begin{array}{c}\text { Revenue } \\
\text { (\$/hr.) }\end{array}$ \\
\hline & Car & $\$ 2.50$ & $\$ 2.20$ & $\$ 2.00$ & $\$ 2.60$ & $\$ 2.80$ & $\$ 2.80$ & & \\
\hline \multirow[t]{3}{*}{1} & Bicycle & $\$ 0.50$ & $\$ 0.70$ & $\$ 0.50$ & $\$ 0.70$ & $\$ 0.60$ & $\$ 0.70$ & $\$ 3,945.27$ & $\$ 778.90$ \\
\hline & Scooter & $\$ 1.00$ & $\$ 1.10$ & $\$ 1.00$ & $\$ 0.90$ & $\$ 1.20$ & $\$ 1.10$ & & \\
\hline & Car & $\$ 2.50$ & $\$ 2.50$ & $\$ 2.30$ & $\$ 2.10$ & $\$ 2.50$ & $\$ 3.50$ & & \\
\hline \multirow[t]{3}{*}{2} & Bicycle & $\$ 0.50$ & $\$ 0.60$ & $\$ 0.60$ & $\$ 0.50$ & $\$ 0.90$ & $\$ 0.50$ & $\$ 3,945.24$ & $\$ 803.97$ \\
\hline & Scooter & $\$ 1.00$ & $\$ 1.00$ & $\$ 1.10$ & $\$ 0.80$ & $\$ 1.30$ & $\$ 0.80$ & & \\
\hline & Car & $\$ 2.50$ & $\$ 1.80$ & $\$ 2.70$ & $\$ 1.50$ & $\$ 3.00$ & $\$ 3.80$ & & \\
\hline \multirow[t]{3}{*}{3} & Bicycle & $\$ 0.50$ & $\$ 0.80$ & $\$ 0.80$ & $\$ 0.50$ & $\$ 0.60$ & $\$ 0.50$ & $\$ 3,945.58$ & $\$ 798.87$ \\
\hline & Scooter & $\$ 1.00$ & $\$ 1.20$ & $\$ 1.20$ & $\$ 0.80$ & $\$ 0.90$ & $\$ 1.00$ & & \\
\hline & Car & $\$ 3.50$ & $\$ 2.50$ & $\$ 2.50$ & $\$ 2.50$ & $\$ 2.50$ & $\$ 2.50$ & & \\
\hline \multirow[t]{3}{*}{4} & Bicycle & $\$ 0.80$ & $\$ 0.60$ & $\$ 0.60$ & $\$ 0.60$ & $\$ 0.60$ & $\$ 0.60$ & $\$ 3,946.29$ & $\$ 835.34$ \\
\hline & Scooter & $\$ 1.20$ & $\$ 0.80$ & $\$ 1.00$ & $\$ 1.00$ & $\$ 1.00$ & $\$ 1.00$ & & \\
\hline & Car & $\$ 3.00$ & $\$ 1.50$ & $\$ 2.80$ & $\$ 1.50$ & $\$ 3.80$ & $\$ 2.50$ & & \\
\hline \multirow[t]{2}{*}{5} & Bicycle & $\$ 0.80$ & $\$ 0.80$ & $\$ 0.80$ & $\$ 0.70$ & $\$ 0.80$ & $\$ 0.70$ & $\$ 3,945.23$ & $\$ 841.36$ \\
\hline & Scooter & $\$ 1.20$ & $\$ 1.00$ & $\$ 0.60$ & $\$ 1.00$ & $\$ 1.20$ & $\$ 1.00$ & & \\
\hline
\end{tabular}

For further analysis, iterations were conducted to the intermediate network. As seen in Table 12 , the users seem to respond and behave accordingly to different pricing policies to minimize and maintain their users' time cost while the company is increasing its revenue. All these results represent the optimal solutions of player 2 , meaning that these are the optimal responses to a pricing policy manually set directly into GAMS.

As seen in Table 12, the company is attempting to increase their revenue, and the pool of network users are minimizing their total time cost by responding accordingly to the strategic price changes of the other player. From these results, some key links should be highlighted; the company's success to increase its revenue is achieved by increasing the price on the links with more usage, such as link 1 and 5. These links are unique in this network since a lot of users will be obligated to use them. Some O-D pairs are dependent only on those links, as seen in A-B, C$\mathrm{A}$, and C-B. 


\section{Sensitivity Analysis}

The best way to understand the global optimum pricing scheme of the system is by evaluating the equilibrium link flow $v_{a}^{m}$ and the parameters that affect the objective function; the free flow travel time $\alpha_{a}^{m}$ and congestion impact coefficient $\beta_{a}^{m}$. This model possesses several dimensions, so it's important to concentrate in one link and mode at a time. The focus is in cars as the mode of transportation to analyze because is the class with higher impact in the system. In addition, this section examines the effects of these parameters on the revenue of the company and time cost of the users. This provides an overall understanding on how this model behaves and how the congestion and time parameters affect driver decision-making.

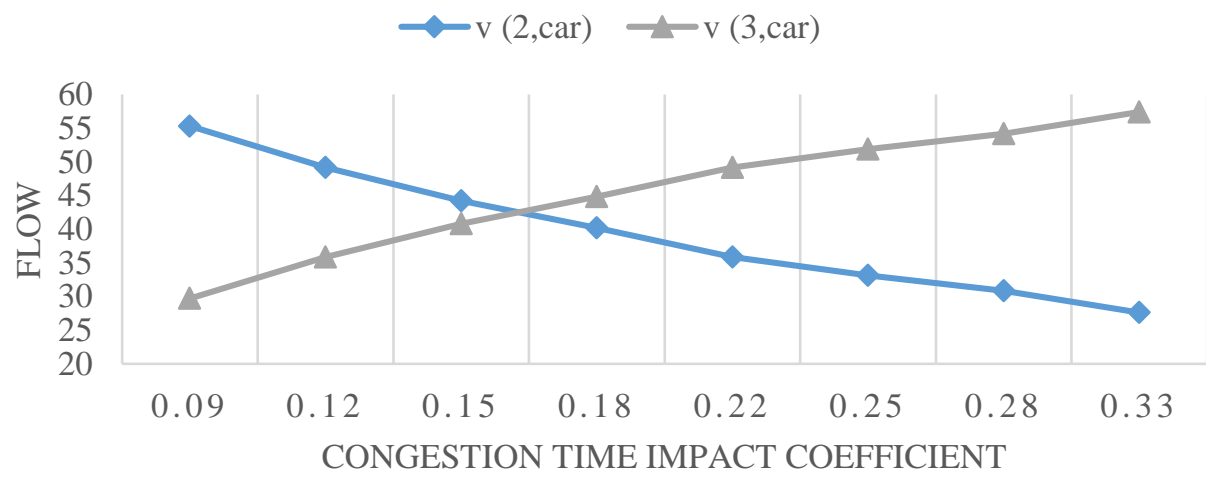

Figure 3. Time impact coefficient $\beta_{a}^{m}$ alteration effect on $v_{a}^{m}$

As seen in Figure 3, a cross-linear behavior can be observed between flows in link 2 and 3. When increasing congestion impact $\beta_{a}^{m}$ in link 2 , more network users would rather take link 3 instead, which is the alternative link option to get from node B to C. In addition, an analysis of the impact of free flow travel time $\alpha_{a}^{m}$ in the same links was performed. As seen in Figure 4, there is a very similar trend on the flow of these two links. The higher the $\alpha_{2}^{c a r}$, the more user networks will be tempted to use link 3 even though congestion could be higher. 


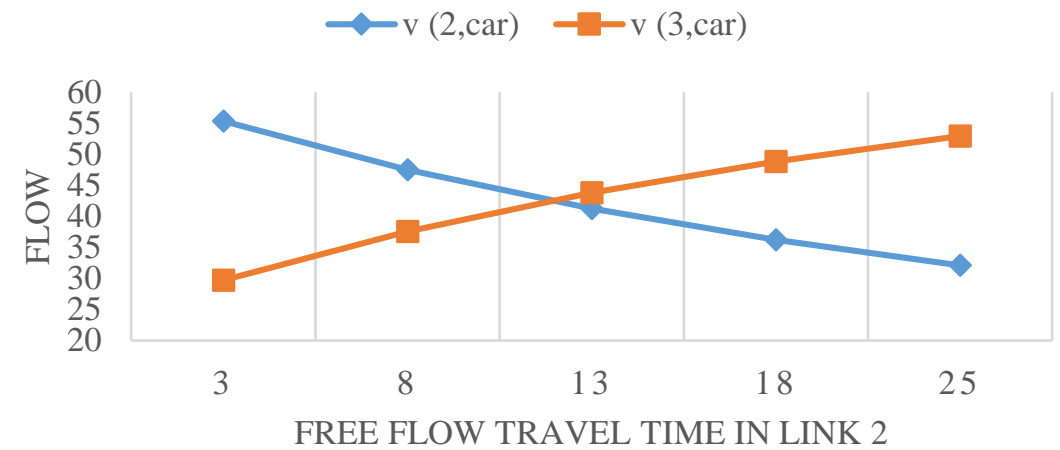

Figure 4. Free flow travel time $\alpha_{a}^{m}$ effects on $v_{a}^{m}$

The impact the price will have on both terms of the objective function was analyzed. This function contains the monetary price paid for the services offered by the shared transport company and the users' time cost of the system, as seen before in the form of eq. F2.

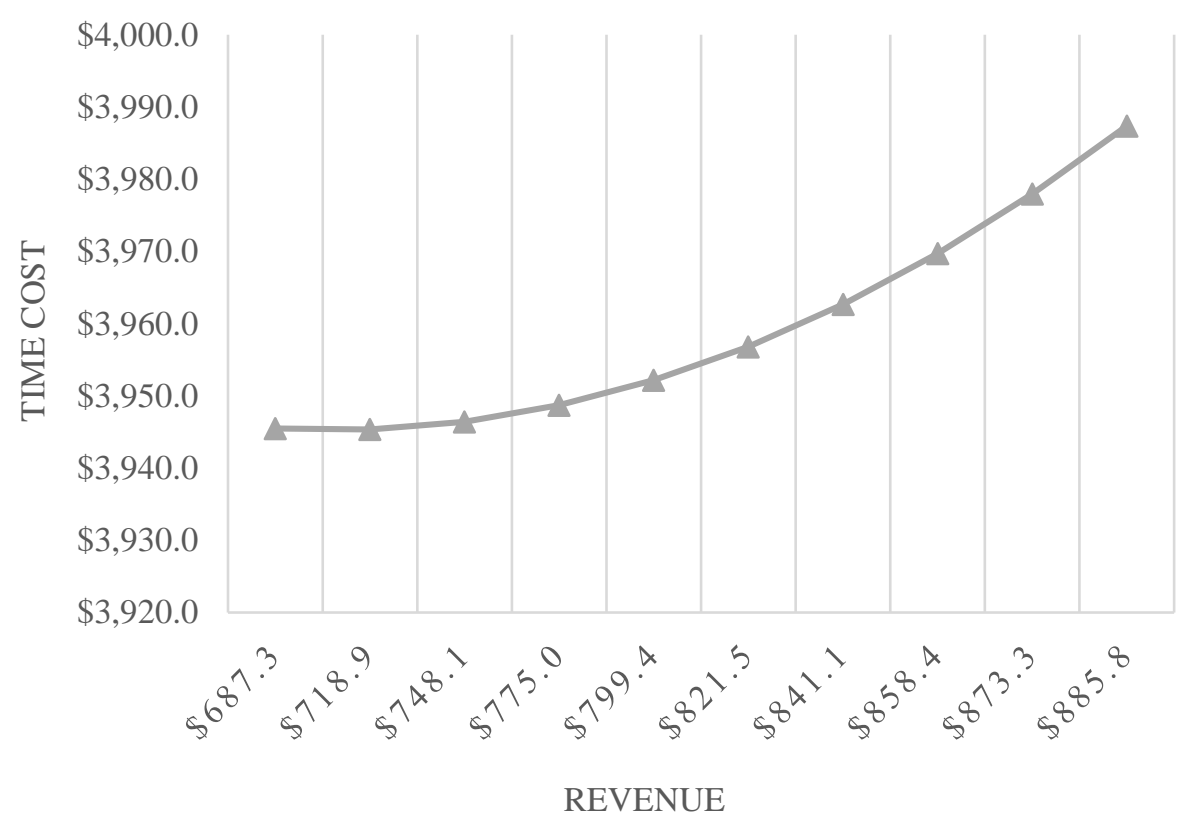

Figure 5. Revenue vs Time cost behavior

As seen in Figure 5, the results of increasing the price on link 3 affect both terms of the objective function. It will naturally increase the revenue since the company is charging more money for a fixed demand. Nevertheless, the users' time cost is increasing by forcing the network users to take routes that will cost them more time. As seen in Figure 6, there is also a relationship between these terms, where the ratio of the revenue over time cost reaches a 
maximum level with the increase of the price charged in link 3 to $\$ 14.10$. At this tipping point, the users' time cost starts to increase while the revenue starts to decrease.

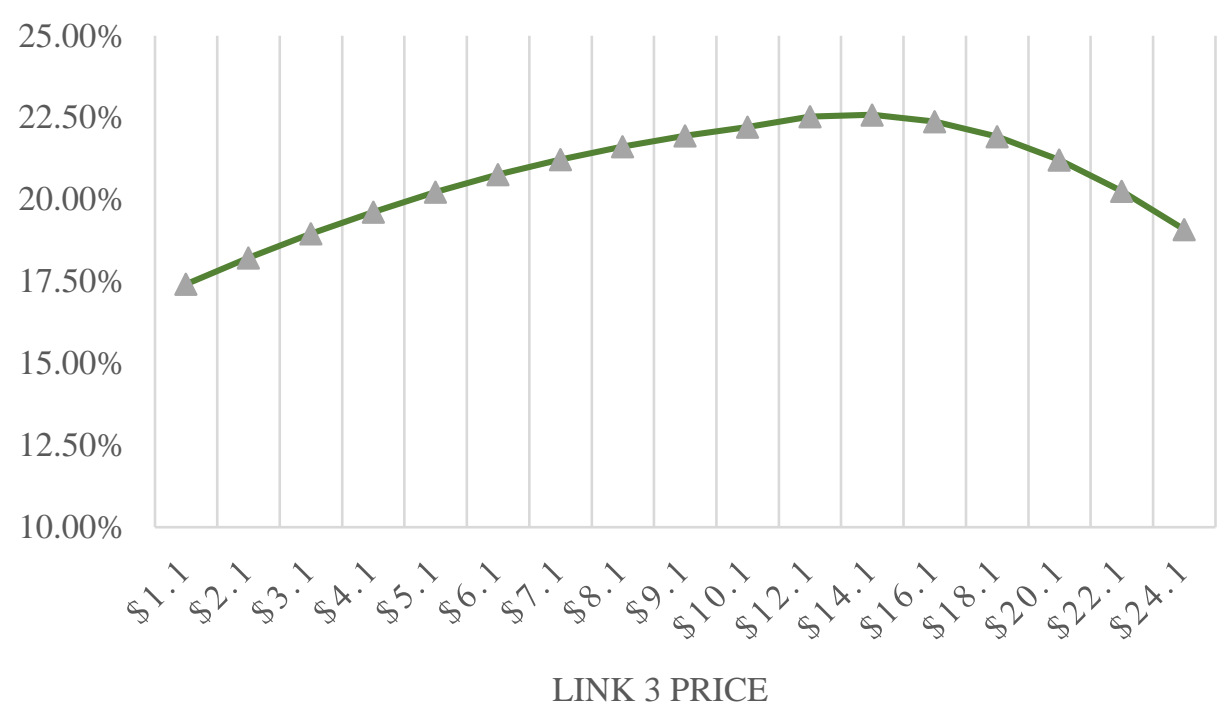

Figure 6. Revenue over Time cost ratio

\section{Conclusions}

This paper examines how to determine optimal price levels for shared transport companies' services in predetermined links. First, this project studied how to establish an optimal pricing policy on given links for hypothetical cases of fixed and elastic demand. For this, both models were developed using a Nash games theoretical framework. These formulations have the objective to maximize company revenue and minimize total users' time cost while considering their route decision-making behavior. Two different network examples were solved using the fixed demand model which determined a system optimum users' flow to minimize users' time cost while proving problem formulation feasibility.

Furthermore, different pricing policy iterations were performed on the intermediate network to better examine the performance of the model. Revenue was increasing while users' time cost seemed to be converging or oscillating within a small range of values. In addition, a sensitivity analysis was conducted on this network to obtain a better overall understanding of the parameters of the model. A crossed-linear behavior of the link flows was expected and found by the controlled alteration of time parameters. Moreover, an analysis of the revenue to time cost ratio 
performance was done. The numerical results confirm how the company can have an impact on both its revenue and time cost by modifying its pricing scheme.

Finally, it should be acknowledged that there are still many variables that could be incorporated into the model to better simulate real-system behavior and constraints. Moreover, it was assumed that all drivers within the network make rational decisions. These models only examined congestion road pricing, but time dependency could be included to better capture rush hours of the network. Further research could incorporate drivers' accidents probabilities and how this could cause further congestion in certain routes of the network.

\section{Acknowledgements}

This project was supported and mentored by Dr. Hongcheng Liu. I also wish to express my gratitude to Yuanbo Wang for his time and help. Finally, I want to thank my family and friends for the unconditional support they have given me throughout my academic years at the University of Florida.

\section{References}

Bell, M. G., \& Cassir, C. (2002). Risk-averse user equilibrium traffic assignment: an application of game theory. Transportation Research Part B: Methodological, 36(8), 671-681. doi: 10.1016/s01912615(01)00022-4

Bertsimas, D., Jaillet, P., \& Martin, S. (2019). Online Vehicle Routing: The Edge of Optimization in Large-Scale Applications. Operations Research, 67(1), 143-162. doi: 10.1287/opre.2018.1763

May, A., \& Milne, D. (2000). Effects of alternative road pricing systems on network performance. Transportation Research Part A: Policy and Practice, 34(6), 407-436. doi: 10.1016/s0965-8564(99)00015-4

Yan, H., \& Lam, W. H. (1996). Optimal road tolls under conditions of queueing and congestion. Transportation Research Part A: Policy and Practice, 30(5), 319-332. doi: 10.1016/0965-8564(96)00003-1

Yang, H., \& Zhang, X. (2002). Multiclass Network Toll Design Problem with Social and Spatial Equity Constraints. Journal of Transportation Engineering, 128(5), 420-428. doi: 10.1061/(asce)0733947x(2002)128:5(420)

Yang, H., \& Zhang, X. (2003). Optimal Toll Design in Second-Best Link-Based Congestion Pricing. Transportation Research Record: Journal of the Transportation Research Board, 1857(1), 85-92. doi: 10.3141/1857-10

Zheng, N., \& Geroliminis, N. (2016). Modeling and optimization of multimodal urban networks with limited parking and dynamic pricing. Transportation Research Part B: Methodological, 83, 3658. doi: $10.1016 /$ j.trb.2015.10.008 